\title{
A Practical Variant of the Semismooth Newton Method for Frictionless Contact Problems*
}

\author{
Tomoshi MIYAMURA**, Yoshihiro KANNO*** and Makoto OHSAKI **** \\ ** Department of Computer Science, College of Engineering, Nihon University, Koriyama 963-8642 Japan \\ miyamura@cs.ce.nihon-u.ac.jp \\ *** Department of Mathematical Informatics, University of Tokyo, Tokyo 113-8656 Japan \\ kanno@mist.i.u-tokyo.ac.jp \\ **** Department of Architecture and Architectural Engineering, Kyoto University, Kyoto 615-8540 Japan \\ ohsaki@archi.kyoto-u.ac.jp
}

\begin{abstract}
In this paper, a simple solution scheme for frictionless contact problems of linear elastic bodies is proposed. The proposed method is a variant of the semismooth Newton method. The frictionless contact problem, which is discretized using the finite element method with lower-order elements and a node-to-node contact model, is considered, although the proposed method can be extended to a node-to-segment or segment-to-segment contact model. The present method can be implemented by slightly modifying a computer program for the semismooth Newton method. In the iterative loop of the proposed method, a symmetric linear structural problem with multi-point constraints is solved. Therefore, the proposed method can be applied to large-scale contact problems. Good convergence of the proposed method compared to the semismooth Newton method is demonstrated in some examples.
\end{abstract}

Key words: Frictionless Contact Problem, Finite Element Method, Semismooth Newton Method

\section{Introduction}

The contact problem is one of the key issues in practical finite element structural analyses. Many researchers have investigated this topic (see, e.g., Refs. (1)(2)). When elastic bodies are discretized using the finite element method and the contact problem is modeled by a node-to-node or node-to-segment contact model, the problem becomes finding all pairs of nodes and/or pairs of node and segment that are in contact. A pair in contact is called an active pair, and the set of all active pairs is referred to as an active set. Conversely, a pair that is not in contact is called an inactive pair, and the set of all inactive pairs is referred to as an inactive set. See, e.g., Wriggers ${ }^{(1)}$ for various algorithms for solving contact problems.

The family of interior-point methods ${ }^{(3)}$ has been shown to be effective for solving convex quadratic programming problems. Such methods can be applied for solving contact problems. One advantage of interior-point methods is that the number of arithmetic operations is bounded by the polynomial of the problem size. Tanoh et al. ${ }^{(4)}$ proposed an interior-point algorithm that can be applied to large-scale contact problems. Comparisons of several numerical methods for frictional contacts were performed by Christensen et al. ${ }^{(5)}$. They applied the semismooth Newton method and the interior-point method to linear elastic contact problems with Coulomb friction and found the semismooth Newton method to be superior. In the case of a sufficiently small friction coefficient, they also proved the global 
convergence property of the semismooth Newton method with a line search using an appropriate merit function. Another strategy proposed for solving quadratic programming problems is the primal-dual active set strategy, which can also be applied to frictionless contact problems $^{(6)}$. Hintermüller et al. ${ }^{(7)}$ showed that the primal-dual active set strategy can be classified as a special form of the semismooth Newton method and attains global convergence in problems with a particular type of coefficient matrix. However, this particular matrix is not exactly the same as the stiffness matrix derived in the finite element formulation. The primal-dual active set approach is equivalent to the trial-and-error method, in which an iterative correction of the active set is conducted until convergence to a solution.

The authors ${ }^{(8)}$ previously proposed a combined interior-point and semismooth Newton method for frictionless contact problems. This method has the advantages of both the interior-point and semismooth Newton methods. A numerical example that is difficult to solve using the semismooth Newton method was found, and this example was solved effectively by the combined method. The performance of the interior-point method was also found to be better than that of the semismooth Newton method. However, one disadvantage of the interior-point method is that a particular preconditioner is necessary when an iterative solver is applied to a linear problem that is solved in the iterative loop of the method, because the coefficient matrix of the linear problem is similar to that of the penalty method. However, semismooth Newton methods have an internal iterative loop for solving a linear structural problem with multi-point constraints (MPCs), which can be solved using a preconditioned conjugate projected gradient method. Recently, efficient solvers based on domain decomposition methods (DDM) have been proposed for solving linear structural problems with MPCs ${ }^{(9)-(12)}$. Therefore, semismooth Newton methods for contact problems can be easily applied to large-scale parallel computations.

In this paper, a simple solution scheme for frictionless contact problems of linear elastic bodies is proposed. The proposed method is a variant of the semismooth Newton method. The formulation of a frictionless contact problem is briefly shown in Section 2. In Section 3 , formulation of a semismooth Newton method is introduced. In Section 4, a practical variant of the semismooth Newton method is proposed. In Section 5, illustrative examples are presented and the performances of the semismooth Newton method and the proposed method are compared. Concluding remarks are presented in Section 6.

\section{Formulation of frictionless contact problems}

The formulation of the two-body contact problem is briefly described in this section. Only the frictionless contact problem is considered here. Each body is discretized using the finite element method with lower-order elements. The simplest node-to-node contact model is adopted; that is, pairs of matching nodes exist on the contact surfaces. However, the discussion in the present paper can be easily extended to a node-to-segment or segment-to-segment contact model (see, e.g., Ref. (1)).

Let $n_{\mathrm{d}}$ denote the number of degrees of freedom (DOFs), and let $n_{\mathrm{C}}$ denote the number of pairs of contact candidate nodes. The stiffness matrix is represented by $\mathbf{K} \in \mathbb{R}^{n_{\mathrm{d}} \times n_{\mathrm{d}}}$. The vector of external loads is denoted by $\mathbf{f} \in \mathbb{R}^{n_{\mathrm{d}}}$, and the nodal displacement vector is denoted by $\mathbf{u} \in \mathbb{R}^{n_{\mathrm{d}}}$. Let $r_{i}$ denote the contact force at the $i$ th pair of contact candidate nodes. The contact force vector $\mathbf{r}=\left[\begin{array}{lll}r_{1} & \cdots & r_{n_{\mathrm{C}}}\end{array}\right]^{\mathrm{T}} \in \mathbb{R}^{n_{\mathrm{c}}}$ is transformed into global coordinates by the transformation matrix $\mathbf{T} \in \mathbb{R}^{n_{\mathrm{c}} \times n_{\mathrm{d}}}$. The equilibrium equation is represented as follows:

$$
\mathbf{K u}-\mathbf{T}^{\mathrm{T}} \mathbf{r}=\mathbf{f} .
$$

Let $h_{i}$ denote the initial gap at the $i$ th pair of contact candidate nodes. The initial gap vector $\mathbf{h}$ is defined as $\mathbf{h}=\left[\begin{array}{lll}h_{1} & \cdots & h_{n_{\mathrm{C}}}\end{array}\right]^{\mathrm{T}} \in \mathbb{R}^{n_{\mathrm{c}}}$. The gap vector $\mathbf{g}$ is defined as $\mathbf{g}=\left[\begin{array}{lll}g_{1} & \cdots & g_{n_{\mathrm{C}}}\end{array}\right]^{\mathrm{T}} \in \mathbb{R}^{n_{\mathrm{c}}}$ and can be represented using the matrix $\mathbf{T}$ in Eq. (1) and the 
vector $\mathbf{h}$, as follows:

$$
\mathbf{g}=-\mathbf{T u}+\mathbf{h} .
$$

For $g_{i}$ and $r_{i}$, the following conditions should be satisfied:

$$
\begin{aligned}
& r_{i} \leq 0, \quad\left(i=1, \cdots, n_{\mathrm{C}}\right), \\
& g_{i} \geq 0, \quad\left(i=1, \cdots, n_{\mathrm{C}}\right), \\
& r_{i} g_{i}=0, \quad\left(i=1, \cdots, n_{\mathrm{C}}\right) .
\end{aligned}
$$

Equation (3) requires that the contact force is not positive. Equation (4) represents the non-penetration condition. The $i$ th pair of contact candidate nodes is considered active when the non-penetration constraint of the pair is satisfied in equality, i.e., $g_{i}=0$ is satisfied. The pair is referred to as inactive when the constraint is inactive, i.e., $g_{i}>0$ is satisfied. The active set is defined as a set of active pairs of the contact candidate nodes. Equation (5), which is called the complementarity condition, requires the contact force to vanish if the gap is positive. Since $r_{i}$ plays the role of the Lagrange multiplier for the non-penetration condition of the $i$ th pair, $\mathbf{r}$ is referred to as the Lagrange multiplier vector in the following sections.

\section{Formulations of the semismooth Newton method}

The frictionless contact problem is written as Eqs. (1)-(5), but instead of Eqs (3)-(5), the following condition is introduced:

$$
\min \left\{-r_{i}, C g_{i}\right\}=0 \quad\left(i=1, \cdots n_{\mathrm{c}}\right)
$$

where $C$ is a positive scaling parameter between $-r_{i}$ and $g_{i}$. Christensen ${ }^{(13)}$ suggested a guideline for selecting the parameter $C$. According to our numerical experiments, however, the magnitude of $C$ does not significantly affect the convergence of the semismooth Newton method. Therefore, the parameter $C$ is taken to be 1.0 in all the illustrative examples discussed in Section 5. The function on the left-hand side of Eq. (6) is semismooth. Note that a function $q: \mathbb{R}^{n} \rightarrow \mathbb{R}$ is semismooth at $\overline{\mathbf{x}} \in \mathbb{R}^{n}$ if $q$ is Lipschitz continuous in the neighborhood of $\overline{\mathbf{x}}, q$ is directionally differentiable at $\overline{\mathbf{x}}$, and $q$ satisfies

$$
\lim _{\mathbf{x} \rightarrow \overline{\mathbf{x}}(\mathbf{x} \neq \overline{\mathbf{x}})} \frac{\left\|q^{\prime}(\mathbf{x} ; \mathbf{x}-\overline{\mathbf{x}})-q^{\prime}(\overline{\mathbf{x}} ; \mathbf{x}-\overline{\mathbf{x}})\right\|}{\|\mathbf{x}-\overline{\mathbf{x}}\|}=0,
$$

where $q^{\prime}(\overline{\mathbf{x}} ; \mathbf{d})$ is the directional derivative of $q$ at $\overline{\mathbf{x}}$ along the direction $\mathbf{d}$. From Eq. (7), it can be shown for a semismooth $q$ that the directional derivative $q^{\prime}(\overline{\mathbf{x}} ; \mathbf{x}-\overline{\mathbf{x}})$ can be approximated by any $\mathbf{H} \in \partial q(\mathbf{x})$ (where $\partial q(\mathbf{x})$ is the Clarke generalized Jacobian of $q$ at $\mathbf{x}$ ) for all $\mathbf{x}$ sufficiently close to $\overline{\mathbf{x}}$ (see Theorem 7.4.3 in Ref. (14)). In general, this property implies that $q\left(\mathbf{x}^{k}\right)+\mathbf{H}^{k} \mathbf{d}^{k}$ with $\mathbf{H}^{k} \in \partial q\left(\mathbf{x}^{k}\right)$ can be regarded as the Newton model of $q\left(\mathbf{x}^{k}+\mathbf{d}\right)$ and thus provides the basis of the semismooth Newton method. See Facchinei and Pang ${ }^{(14)}$ for more detail.

Equations (1), (2), and (6) are equivalent to Eqs. (1)-(5). Therefore, the equivalent problem is summarized as follows:

$$
\begin{aligned}
& \mathbf{K u}-\mathbf{T}^{\mathrm{T}} \mathbf{r}=\mathbf{f}, \\
& \mathbf{g}=-\mathbf{T} \mathbf{u}+\mathbf{h}, \\
& \min \left\{-r_{i}, C g_{i}\right\}=0 \quad\left(i=1, \cdots n_{\mathrm{c}}\right) .
\end{aligned}
$$

The system of equations (8) can be solved by a semismooth Newton method ${ }^{(5)}$. The procedure of the semismooth Newton method can be explained as follows. From the strict definition of a semismooth Newton method, the system of nonlinear equations should be solved in order to find the search direction at non-differentiable points of the system of equations (8), because the directional derivative is nonlinear in the search direction at 
non-differentiable points. The min function in Eqs. (8) is non-differentiable when $-r_{i}=C g_{i}$ is satisfied. Here, the directional derivative of $\min \left\{-\Delta r_{i}, C \Delta g_{i}\right\}$ is approximated by $-\Delta r_{i}$ at this non-differentiable point. Then, Eq. (8) can be linearized as follows:

$$
\begin{aligned}
& {\left[\begin{array}{cc}
\mathbf{K} & -\mathbf{T}^{\mathrm{T}} \\
-\mathbf{T}_{\mathrm{A}} & \mathbf{O}
\end{array}\right]\left[\begin{array}{c}
\Delta \mathbf{u} \\
\Delta \mathbf{r}
\end{array}\right]=-\left[\begin{array}{c}
\mathbf{K} \mathbf{u}^{k}-\mathbf{T}^{\mathrm{T}} \mathbf{r}^{k}-\mathbf{f} \\
-\mathbf{T}_{\mathrm{A}} \mathbf{u}^{k}+\mathbf{h}
\end{array}\right],} \\
& \Delta \mathbf{r}_{\mathrm{I}}=-\mathbf{r}_{\mathrm{I}}^{k},
\end{aligned}
$$

where the subscripts I and A indicate the components with respect to the active and inactive pairs, respectively, and the superscript $k$ denotes the iteration counter. The vector $\left[\begin{array}{ll}\Delta \mathbf{u} & \Delta \mathbf{r}\end{array}\right]^{\mathrm{T}}$ represents the search direction. Let $I$ and $A$ be a partition of $\left\{1, \cdots, n_{\mathrm{c}}\right\}$, i.e., $I$ and $A$ are sets satisfying $I \cup A=\left\{1, \cdots, n_{\mathrm{c}}\right\}$ and $I \cap A=\varnothing$. Such a partition is written as $(I, A)$ in the following. The partition at the $k$ th iteration is denoted as $\left(I^{k}, A^{k}\right)$. In the semismooth Newton method, the partition $\left(I^{k}, A^{k}\right)$ is obtained by using Eq. (6), as follows:

$$
\left\{\begin{array}{c}
-r_{i}^{k} \leq C g_{i}^{k} \Rightarrow \text { inactive }\left(i \in I^{k}\right) \\
-r_{i}^{k}>C g_{i}^{k} \Rightarrow \text { active }\left(i \in A^{k}\right)
\end{array} .\right.
$$

By considering Eq. (10) for the inactive components, Eq. (9) can be rewritten as follows:

$$
\left[\begin{array}{cc}
\mathbf{K} & -\mathbf{T}_{\mathrm{A}}^{\mathrm{T}} \\
-\mathbf{T}_{\mathrm{A}} & \mathbf{O}
\end{array}\right]\left[\begin{array}{c}
\Delta \mathbf{u} \\
\Delta \mathbf{r}_{\mathrm{A}}
\end{array}\right]=-\left[\begin{array}{c}
\mathbf{K} \mathbf{u}^{k}-\mathbf{T}_{\mathrm{A}}^{\mathrm{T}} \mathbf{r}_{\mathrm{A}}^{k}-\mathbf{f} \\
-\mathbf{T}_{\mathrm{A}} \mathbf{u}^{k}+\mathbf{h}
\end{array}\right] .
$$

The search direction $\left[\begin{array}{ll}\Delta \mathbf{u} & \Delta \mathbf{r}\end{array}\right]^{\mathrm{T}}$ is obtained from Eqs. (10) and (12). Note that the second line in Eq. (12) is regarded as MPCs, which are written as equality constraints to the linear structural problem in the first line of Eq. (12). The problem can be solved by an efficient serial or parallel solver based on the domain decomposition method (DDM) ${ }^{(9)-(12)}$. The semismooth Newton method can therefore easily be applied to large-scale frictionless contact problems. The solutions are updated as follows:

$$
\left\{\begin{array}{l}
\mathbf{u}^{k+1}=\mathbf{u}^{k}+\alpha^{k+1} \Delta \mathbf{u} \\
\mathbf{r}^{k+1}=\mathbf{r}^{k}+\alpha^{k+1} \Delta \mathbf{r}
\end{array} .\right.
$$

The step length $\alpha^{k+1} \leq 1.0$ is determined by the line search to minimize the following merit function:

$$
\Theta(\mathbf{u}, \mathbf{r})=\sqrt{\mathbf{H}(\mathbf{u}, \mathbf{r})^{\mathrm{T}} \mathbf{H}(\mathbf{u}, \mathbf{r}),}
$$

where

$$
\mathbf{H}(\mathbf{u}, \mathbf{r})=\left[\begin{array}{c}
\mathbf{K u}-\mathbf{T}^{\mathrm{T}} \mathbf{r}-\mathbf{f} \\
{\left[-\min \left\{-r_{i}, C g_{i}\right\}\right]}
\end{array}\right] .
$$

A bisection method is used for this procedure. Although the global convergence property of the semismooth Newton method is ensured by the line search, the number of iterations is not always reduced. The convergence of the iteration is checked using the merit function:

$$
\Theta(\mathbf{u}, \mathbf{r})<\varepsilon_{\text {converge }},
$$

where $\varepsilon_{\text {converge }}$ is a convergence threshold.

\section{A practical variant of the semismooth Newton method}

In this section, a practical variant of the semismooth Newton method is proposed. Although the method is heuristic and not based on a rigorous mathematical background, the convergence of the variant is equivalent to or is considerably better than that of the original semismooth Newton method in the three illustrative examples shown in Section 5. The 
proposed method can be implemented by slightly modifying a computer program of the semismooth Newton method.

In the proposed method, a slightly modified Lagrange multiplier vector is used for condition (11) to detect the active/inactive partition $(I, A)$. The partition derived by using the modified Lagrange multiplier vector is denoted by $(\tilde{I}, \tilde{A})\left(\tilde{I} \cup \tilde{A}=\left\{1, \cdots, n_{\mathrm{c}}\right\}\right.$, $\tilde{I} \cap \tilde{A}=\varnothing)$.

The modified Lagrange multiplier $\tilde{\mathbf{r}}^{k+1}$ at step $k+1$ is defined as follows using the partition $\left(\tilde{I}^{k}, \tilde{A}^{k}\right)$ at step $k$ :

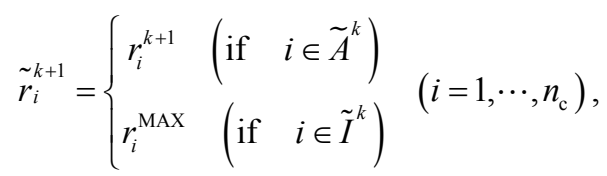

where $r_{i}^{\mathrm{MAX}}$ represents the maximum value of the Lagrange multiplier that pair $i$ has experienced. The value of $r_{i}^{\mathrm{MAX}}$ is equal to or larger than 0.0 when the Lagrange multiplier is initialized as 0.0 in the first step. The modified Lagrange multiplier $\tilde{\mathbf{r}}^{k+1}$ is used only for updating the partition $(\tilde{I}, \tilde{A})$. After the gap vector $\mathbf{g}^{k+1}$ is calculated, the partition is updated using $\tilde{\mathbf{r}}^{k+1}$, as follows:

$$
\left\{\begin{array}{l}
-\tilde{r}_{i}^{k+1} \leq C g_{i}^{k+1} \Rightarrow \text { inactive }\left(i \in \tilde{I}^{k+1}\right) \\
\tilde{r}_{i}^{k+1}>C g_{i}^{k+1} \Rightarrow \text { active }\left(i \in \tilde{A}^{k+1}\right)
\end{array} .\right.
$$

Note that $r_{i}$ in condition (11) is replaced by $\tilde{r}_{i}$ in condition (18).

If the Lagrange multiplier for an active pair becomes positive, the status of the pair is changed from active to inactive, and the value of $r_{i}^{\mathrm{MAX}}$ is updated to a positive value. When $i \in \tilde{I}^{k}$ and $r_{i}^{\mathrm{MAX}}$ is a positive value, the left-hand side of inequality in the second line of (18) for the pair $i$ is negative. Therefore, inequality in the second line of (18) is satisfied only when the gap $g_{i}^{k+1}$ is negative and $\left|g_{i}^{k+1}\right|$ is a moderately large value; that is, inequality in the second line of (18) is satisfied when large penetration occurs. In another words, once a pair of contact candidate nodes experiences the active state and changes to an inactive state from the active state, it is difficult for the pair to have an active state again. This property prevents oscillation between the active and inactive states. It is also noted that the effect of the modified Lagrange multiplier depends on the scaling parameter $C$. However, the parameter $C$ is taken to be 1.0 in all the illustrative examples discussed in Section 5 .

The search direction $\left[\begin{array}{ll}\Delta \mathbf{u} & \Delta \mathbf{r}\end{array}\right]^{\mathrm{T}}$ is obtained from Eqs. (19) and (20), as follows:

$$
\begin{aligned}
& {\left[\begin{array}{cc}
\mathbf{K} & -\mathbf{T}_{\tilde{\AA}}^{\mathrm{T}} \\
-\mathbf{T}_{\tilde{\AA}} & \mathbf{O}
\end{array}\right]\left[\begin{array}{l}
\Delta \mathbf{u} \\
\Delta \mathbf{r}_{\tilde{\AA}}
\end{array}\right]=-\left[\begin{array}{c}
\mathbf{K} \mathbf{u}^{k}-\mathbf{T}_{\tilde{\AA}}^{\mathrm{T}} \mathbf{r}_{\tilde{A}}^{k}-\mathbf{f} \\
-\mathbf{T}_{\tilde{\AA}} \mathbf{u}^{k}+\mathbf{h}
\end{array}\right],} \\
& \Delta \mathbf{r}_{\tilde{\mathrm{I}}}=-\mathbf{r}_{\tilde{I}}^{k},
\end{aligned}
$$

where the subscripts $\tilde{\mathrm{I}}$ and $\tilde{\mathrm{A}}$ indicate the components that are included in the sets $\tilde{I}$ and $\tilde{A}$, respectively. The solutions are updated using Eq. (13). Since Eq. (19) is similar to Eq. (12), Eq. (19) can also be solved by the domain decomposition methods (DDM) for a linear structural problem with MPCs ${ }^{(9)-(12)}$. Therefore, the proposed method can also be applied to large-scale frictionless contact problems.

The convergence of the iteration is checked using Eq. (16), in which the merit function is calculated using the original partition $\left(I^{k+1}, A^{k+1}\right)$, the Lagrange multiplier $\mathbf{r}$ and the gap g. Note that the partition $\left(I^{k+1}, A^{k+1}\right)$ is obtained by using condition (11). When Eq. (16) is satisfied, partition $\left(I^{k+1}, A^{k+1}\right)$ and partition $\left(\tilde{I}^{k+1}, \tilde{A}^{k+1}\right)$ are compared. Iteration is continued if partition $\left(I^{k+1}, A^{k+1}\right)$ and partition $\left(\tilde{I}^{k+1}, \tilde{A}^{k+1}\right)$ are different. In the illustrative examples shown in Section 5 , however, partition $\left(\tilde{I}^{k+1}, \tilde{A}^{k+1}\right)$ agrees with partition $\left(I^{k+1}, A^{k+1}\right)$ when Eq. (16) is satisfied for the first time. Note that the line search 
with merit function in Eq. (14) does not contribute to the convergence, according to our numerical experiments. The algorithms of the original and modified semismooth Newton methods are summarized in Tables 1 and 2, respectively.

\section{Illustrative examples}

In the following examples, Young's modulus and Poisson's ratio are taken to be 6.0 $\mathrm{MPa}$ and 0.27 , respectively. The convergence threshold $\varepsilon_{\text {converge }}$ in Eq. (16) is equal to $1.0 \times 10^{-7}$. In the figures, the semismooth Newton method and the proposed method are denoted by SSN and MSSN, respectively. In the implementation of the proposed method, the open source sparse solver SPOOLES ${ }^{(15)}$ is employed for solving the systems of linear equations in Eq. (12).

Table 1: Algorithm of the semismooth Newton method.

Initialize:

Assign $C$ in Eqs. (11) (let $C=1$ in this study)

Set $\mathbf{u}^{0}$ and $\mathbf{r}^{0}$ (let $\mathbf{u}^{0}=\mathbf{r}^{0}=\mathbf{0}$ in this study)

Obtain the partition $\left(I^{0}, A^{0}\right)$ from $\mathbf{r}^{0}$ and $\mathbf{g}^{0}$ (Eq. (11))

Iterate $k=0,1,2, \cdots$ until convergence:

Solve Eq. (12) to obtain the search direction $\Delta \mathbf{u}$ and $\Delta \mathbf{r}_{\mathrm{A}}$

Set $\Delta \mathbf{r}_{\mathrm{I}}$ using Eq. (10)

Calculate $\alpha^{k+1}$ by the line search to minimize the merit function in Eq. (14)

Update $\left\{\begin{array}{l}\mathbf{u}^{k+1}=\mathbf{u}^{k}+\alpha^{k+1} \Delta \mathbf{u} \\ \mathbf{r}^{k+1}=\mathbf{r}^{k}+\alpha^{k+1} \Delta \mathbf{r}\end{array}\right.$ (Eq. (13))

Obtain the partition $\left(I^{k+1}, A^{k+1}\right)$ from $\mathbf{r}^{k+1}$ and $\mathbf{g}^{k+1}$ (Eq. (11))

Check convergence using Eq. (16)

Table 2: Algorithm of the proposed variant of semismooth Newton method.

Initialize:

Assign $C$ in Eqs. (11) and (18) (let $C=1$ in this study)

Let $\mathbf{u}^{0}=\mathbf{r}^{0}=\mathbf{0}$, and $\tilde{\mathbf{r}}^{0} \equiv \mathbf{r}^{0}$

Obtain the partition $\left(\tilde{I}^{0}, \tilde{A}^{0}\right)$ from $\tilde{\mathbf{r}}^{0}$ and $\mathbf{g}^{0}$ (Eq. (18))

Iterate $k=0,1,2, \cdots$ until convergence:

Solve Eq. (19) to obtain the search direction $\Delta \mathbf{u}$ and $\Delta \mathbf{r}_{\tilde{A}}$

Set $\Delta \mathbf{r}_{\tilde{I}}$ using Eq. (20)

Update $\left\{\begin{array}{l}\mathbf{u}^{k+1}=\mathbf{u}^{k}+\Delta \mathbf{u} \\ \mathbf{r}^{k+1}=\mathbf{r}^{k}+\Delta \mathbf{r}_{\tilde{\AA}}\end{array} \quad\right.$ (similar to Eq. (13) with $\alpha^{k+1}=1.0$ )

Obtain $\tilde{\mathbf{r}}^{k+1}$ using Eqs. (17)

Obtain the partition $\left(\tilde{I}^{k+1}, \tilde{A}^{k+1}\right.$ ) from $\tilde{\mathbf{r}}^{k+1}$ and $\mathbf{g}^{k+1}$ (Eq. (18))

Obtain the partition $\left(I^{k+1}, A^{k+1}\right.$ ) from $\mathbf{r}^{k+1}$ and $\mathbf{g}^{k+1}$ (Eq. (11))

Check convergence using Eq. (16) (the merit function is calculated using $\mathbf{r}^{k+1}, \mathbf{g}^{k+1}$, and the partition $\left.\left(I^{k+1}, A^{k+1}\right)\right)$

If $\Theta\left(\mathbf{u}^{k+1}, \mathbf{r}^{k+1}\right)<\varepsilon_{\text {converge }}$

If the partition $\left(I^{k+1}, A^{k+1}\right)$ and the partition $\left(\tilde{I}^{k+1}, \tilde{A}^{k+1}\right)$ are the same Stop iteration

Else

Set the partition $\left(I^{k+1}, A^{k+1}\right)$ as the partition $\left(\tilde{I}^{k+1}, \tilde{A}^{k+1}\right)$ and continue iteration 


\subsection{Pair of plates with simple supports}

A pair of plates with simple supports, as shown in Fig. 1, is considered. This model was solved by the combined interior-point and semismooth Newton method in Ref. (8). The numbers of divisions in the $x$ and $z$ directions of each plate are five and four, respectively. Four models with the following numbers of subdivisions in the $y$ direction are analyzed: 100, 200, 300, and 400. The sizes of the two plates are shown in Fig. 1. The lateral nodal displacements in the $-z$ direction are uniformly prescribed on the upper surface of the upper plate. The bottom surface of the lower plate is simply supported; that is, two edges at $y=0$ and $10 \mathrm{~m}$ of the lower surface of the lower plate are supported by pins. The lower surface of the upper plate and the upper surface of the lower plate are initially in contact. The mesh patterns of the two surfaces are the same, and the initial gaps at each pair of nodes are zero. After the prescribed displacements are applied, pairs of nodes around the two supports are in contact and the other pairs are not in contact. Figure 2 shows the convergence properties of the semismooth Newton method with and without the line search and the present modified method. The number of iterations of the proposed method is about $50 \%$ to $56 \%$ of that of the semismooth Newton method with the line search.

This problem is solved with considerably fewer iterations using the interior-point method or combined interior-point and semismooth Newton method proposed previously by the present authors ${ }^{(8)}$ than the semismooth Newton method. However, the linear problem that is solved in the iteration of the interior-point method is not represented as a linear structural problem with MPCs, as in Eq. (19). Therefore, it is difficult to immediately combine these methods with DDM.

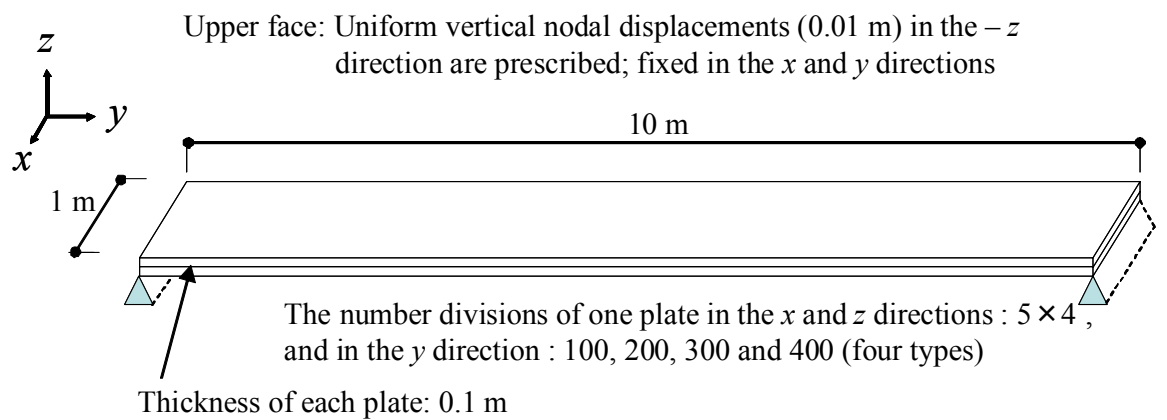

Fig. 1: Analysis model of a pair of plates with simple supports.

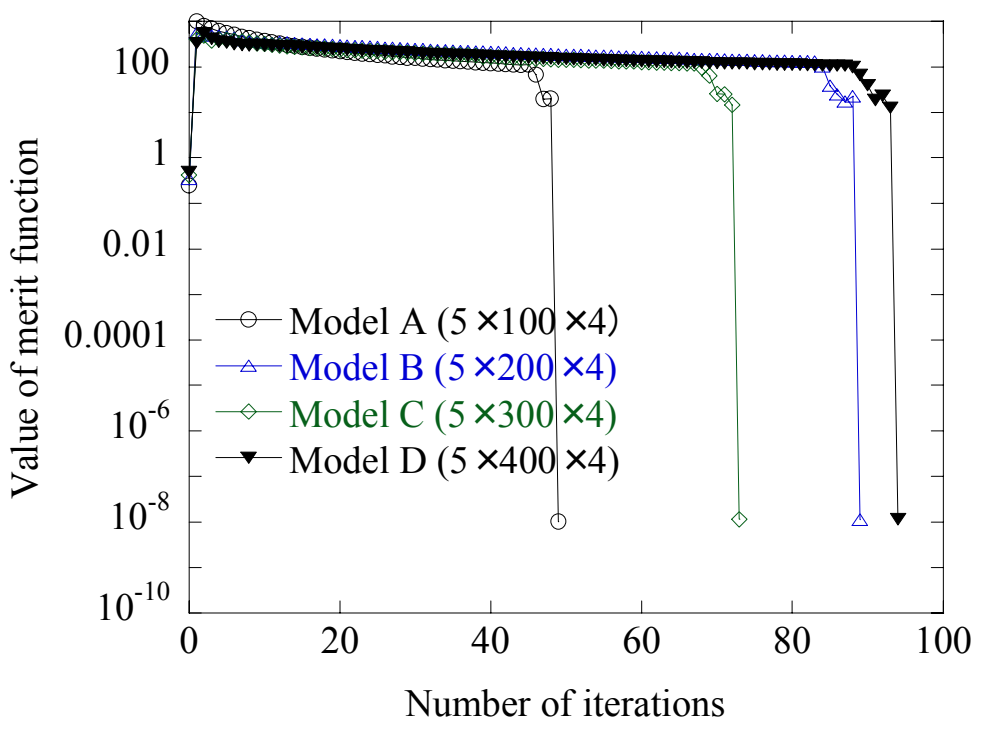

(a) SSN without line search 


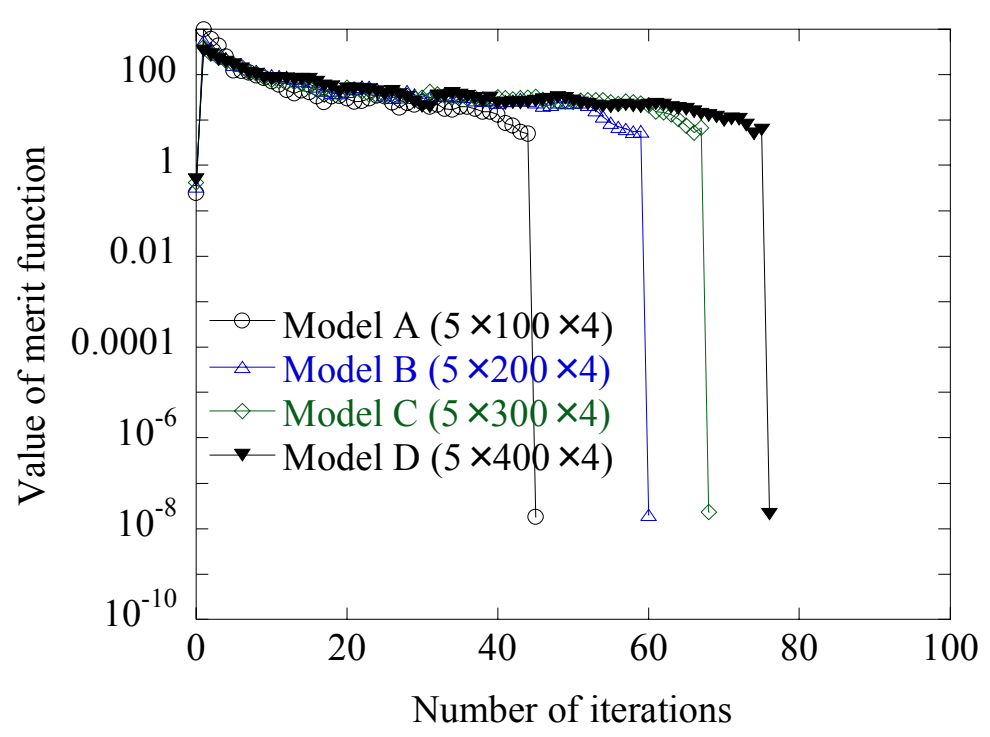

(b) SSN with line search

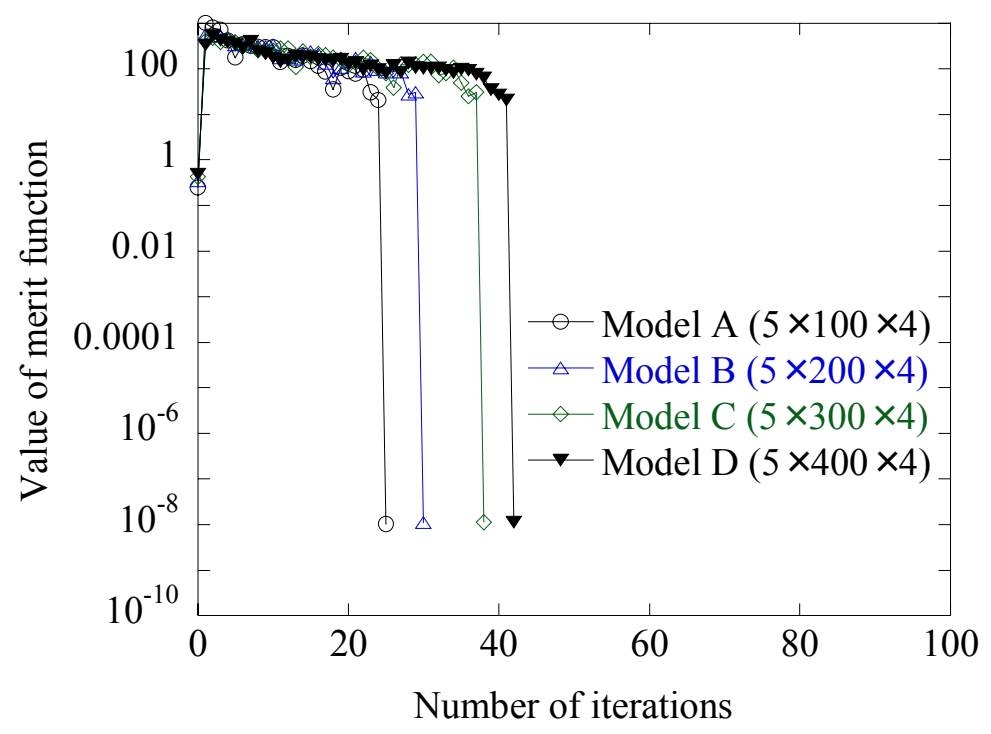

(c) MSSN

Fig. 2: Convergence history of $\Theta$ (pair of plates with simple supports).

\subsection{Pair of plates supported at one side}

This illustrative example is similar to that discussed in Section 5.1. The pair of plates shown in Fig. 3 is considered. The sizes of the plates are shown in the figure. The numbers of divisions of each plate in the $x, y$, and $z$ directions are 10,60 , and 4 , respectively. The total number of elements of the two plates is 4,800 . The total number of nodes is 6,710 and 
the total number of DOFs is 20,130 . The lateral nodal displacements in the $-z$ direction are uniformly prescribed on the upper surface of the upper plate, where the displacements in the $x$ and $y$ directions are fixed. Some nodes on the bottom surface of the lower plate are fixed, as shown in Fig. 3. The lower surface of the upper plate and the upper surface of the lower plate are initially in contact. The mesh patterns of the two surfaces are the same, and the magnitude of initial gap at each pair of nodes is zero. After the prescribed displacements are applied, the pairs of nodes around the fixed area remain in contact, and the other pairs are inactive. Figure 4 shows the convergence properties of the semismooth Newton method and the present modified method. The number of iterations of the proposed method is about $54 \%$ of that of the semismooth Newton method without the line search.

It is also noted that the number of iterations of the semismooth Newton method with the line search is larger than that of the semismooth Newton method without the line search in this example. As described in Section 3, the global convergence property of the semismooth Newton method is ensured by the line search, although the number of iterations is not always reduced by the line search.

\subsection{Contact between flat surface and curved surface defined by a sinusoidal wave}

In this illustrative example, the contact between a flat surface and a curved surface is considered. As shown in Fig. 5, two solids are in contact. The lower surface of the upper solid is flat, whereas the upper surface of the lower solid is represented by a sinusoidal function. The sizes are indicated in the figure. Note that the gap in the initial configuration is magnified in the figure. The gap $g^{\text {init }}$ in the initial configuration, which is a function of $y$, is calculated as follows:

$$
g^{\text {init }}(y)=0.1[1-\sin (\pi y / 40)] \quad(0 \leq y \leq 40) .
$$

In Eq. (21), $y$ is assumed to be 0.0 at the left face in Fig. 5. The numbers of divisions of each solid in the $x, y$, and $z$ directions are 10, 40, and 10, respectively. The mesh pattern of the curved surface projected on the $x y$ plane is the same as the mesh pattern of the flat surface; that is, the curved surface is made by moving the $z$ coordinates of the corresponding flat surface. Therefore, the node-to-node contact model can be used if the gap is sufficiently small. The total number of elements for the two solids is 8,000 . The total number of nodes is 9,922 , and the total number of DOFs is 29,766 .

The lateral nodal displacements in the $-z$ direction, the magnitude of which is $0.01 \mathrm{~m}$, are uniformly prescribed on the upper surface of the upper solid, where the displacements in the $x$ and $y$ directions are fixed. All nodes on the bottom surface of the lower solid are fixed. After the prescribed displacements are applied, the pairs of nodes around the central area come into contact. Note that this example is a typical case of bodies with complex geometries that are in contact. In this case, the semismooth Newton method with or without the line search converges very quickly. As shown in Fig. 6, the convergence of the proposed method is the same as that of the semismooth Newton method without the line search. Note that the semismooth Newton method may be effective for many problems of bodies with complex geometries. The results of the illustrative example in this section show that for such problems, the convergence of the proposed method is similar to that for the semismooth Newton method. By contrast, the semismooth Newton method is not effective for the problems described in Sections 5.1 and 5.2. This provided the motivation to develop the proposed method. 
Upper face: Uniform vertical nodal displacements $(0.01 \mathrm{~m})$ in the $-z$ direction are prescribed; fixed in the $x$ and $y$ directions

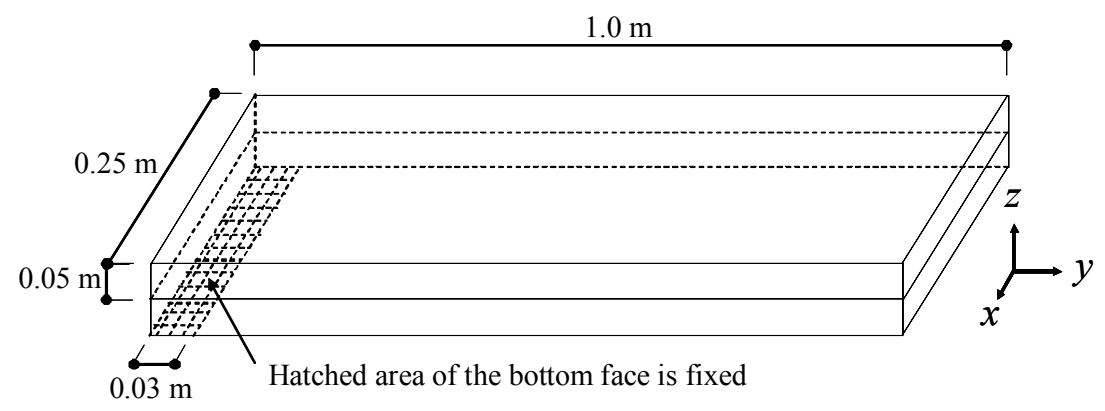

The number of divisions in the $x, y$, and $z$ directions of one plate: $10 \times 60 \times 4$

Fig. 3: Analysis model of a pair of plates supported at one side.

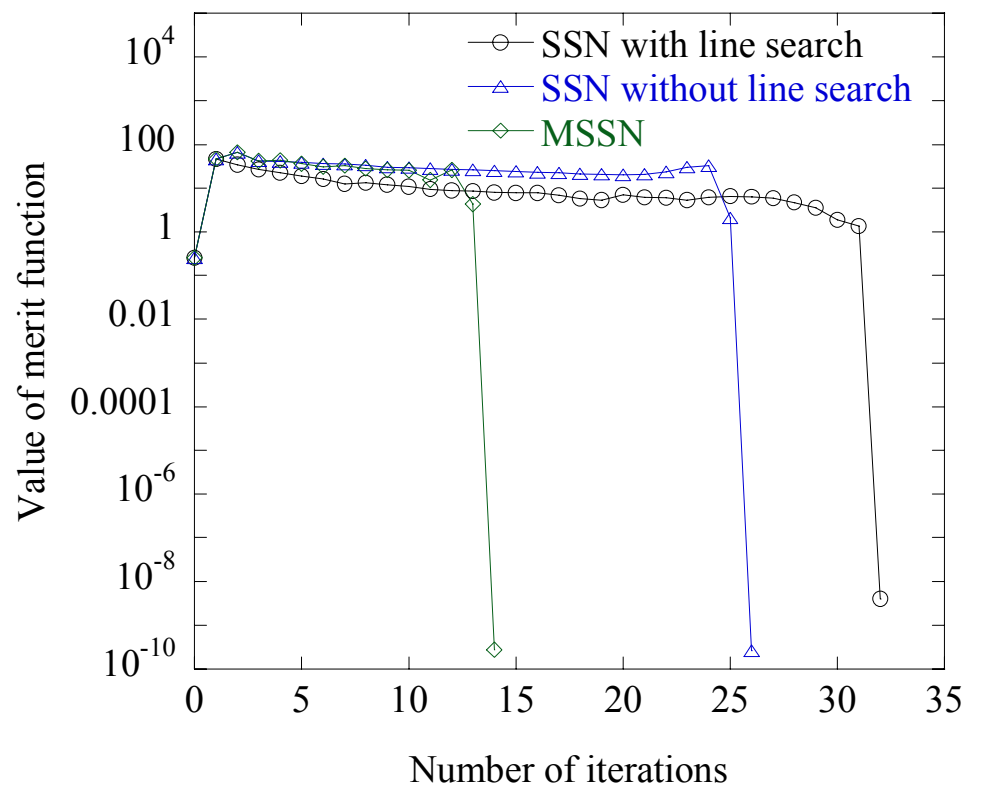

Fig. 4: Convergence history of $\Theta$ (model of a pair of plates supported on one side).

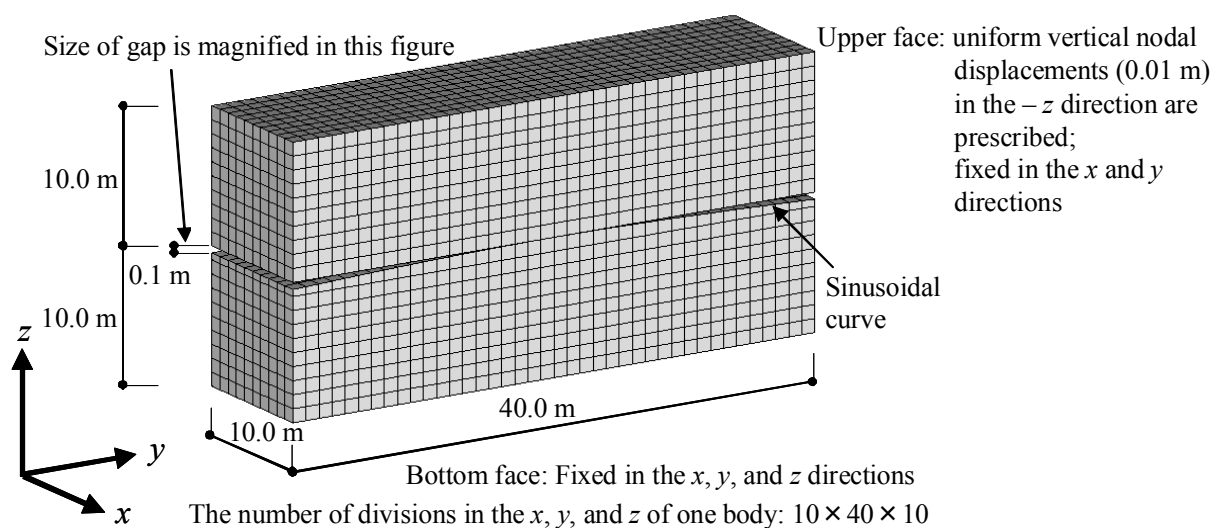

Fig. 5: Contact between the flat and curved surfaces. 


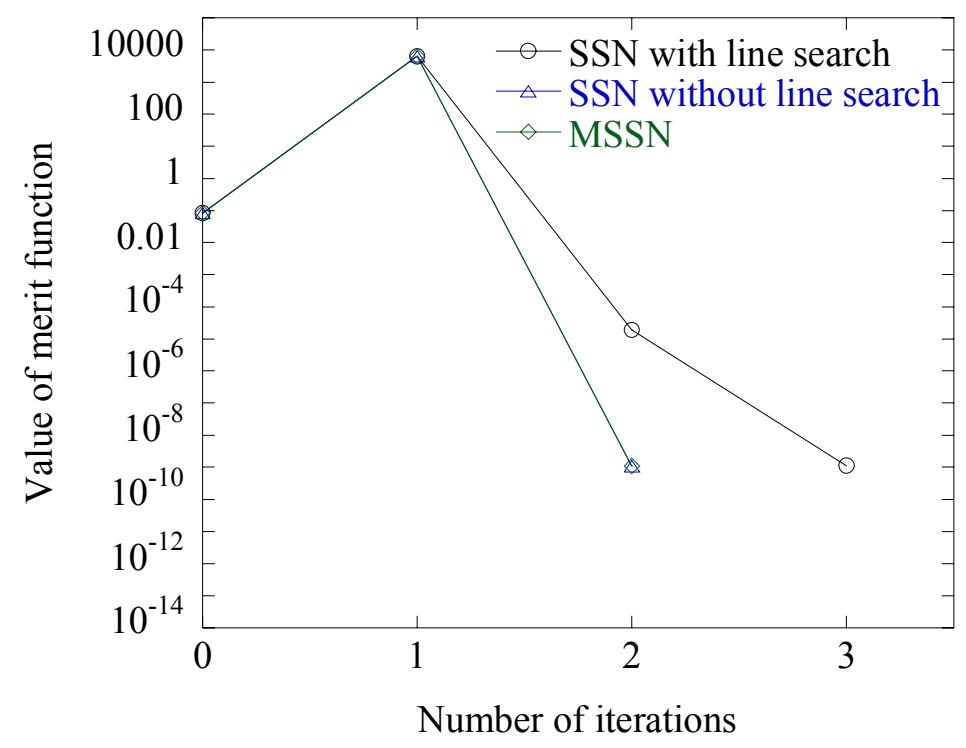

Fig. 6: Convergence history of $\Theta$ (contact between the flat and the curved surfaces).

\section{Concluding remarks}

A simple solution scheme for frictionless contact problems of linear elastic bodies was proposed. The proposed method is a variant of the semismooth Newton method, which can be implemented by slightly modifying a computer program for the semismooth Newton method. In the iterative loop of the proposed method, a symmetric linear problem with MPCs, which can be solved by the conjugate projected gradient method, was solved, indicating that the proposed method can be applied to large-scale contact problems. For illustrative examples in which the convergence of the semismooth Newton method was not very good, the convergence of the proposed method was considered considerably better than the that of the semismooth Newton method. In an example in which the semismooth Newton method converged within a few iterations, the convergence of the proposed method was the same as that of the semismooth Newton method

\section{Acknowledgements}

In this study, the first author was supported in part by a Grant-In-Aid for Scientific Research C (20560061) from JSPS. The first author was also supported in part by a grant from MEXT of Japan to promote multidisciplinary research projects at the College of Engineering, Nihon University.

\section{References}

(1) Wriggers, P., Computational Contact Mechanics (2nd ed.), (2006), Springer-Verlag, Berlin, Germany.

(2) Laursen T.A., Computational Contact and Impact Mechanics, (2003), Springer-Verlag, Berlin, Germany.

(3) Ye Y., Interior-Point Algorithms: Theory and Analysis, (1997), John Wiley \& Sons, New York, NY. 
(4) Tanoh G.., Renardy Y. and Noll D., Computational experience with an interior point algorithm for large scale contact problems, Technical Report, Laboratoire MIP, Université Paul Sabatier, Toulouse, 04-22 (2004), available from Optimization Online: $<$ http://www.optimization-online.org/DB_HTML/2004/12/1012.html>

(accessed 2008-8-16).

(5) Christensen P.W., Klarbring A., Pang J.S. and Stromberg N., Formulation and comparison of algorithms for frictional contact problems, International Journal for Numerical Methods in Engineering, Vol. 42 (1998), pp.145-173.

(6) Hüeber S. and Wohlmuth B., A primal-dual active set strategy for non-linear multibody contact problems, Computer Methods in Applied Mechanics and Engineering, Vol. 194 (2005), pp. 3147-3166.

(7) Hintermüller M., Ito K. and Kunisch K., The primal-dual active set strategy as a semismooth Newton method, SIAM Journal on Optimization, Vol. 13 (2003), pp. 865-888.

(8) Miyamura T., Kanno Y. and Ohsaki M., Combination of Interior-Point Method and Semismooth Newton Method for Large-Scale Frictionless Contact Problems, Proceedings of the 8th World Congress on Computational Mechanics (WCCM8) / 5th European Congress on Computational Methods in Applied Sciences and Engineering (ECCOMAS2008), (2008-6, 7), (CD-ROM).

(9) Farhat C., Lacour C., Rixen D., Incorporation of linear multipoint constraints in substructure based iterative solvers. Part 1: A numerically scalable algorithm, International Journal for Numerical Methods in Engineering, Vol. 43 (1998), pp. 997-1016.

(10) Rixen D., Extended preconditioners for the FETI method applied to constrained problems, International Journal for Numerical Methods in Engineering, Vol. 54 (2002), pp. $1-26$.

(11) Miyamura T., Incorporation of multipoint constraints into the balancing domain decomposition method and its parallel implementation, International Journal for Numerical Methods in Engineering, Vol. 69 (2007), pp. 326-346.

(12) Bavestrello H., Avery P. and Farhat C., Incorporation of linear multipoint constraints in domain-decomposition-based iterative solvers - Part II: Blending FETI-DP and mortar methods and assembling floating substructures, Computer Methods in Applied Mechanics and Engineering, Vol. 196, No. 8 (2007), pp. 1347-1368.

(13) Christensen, P.W., A semi-smooth Newton method for elasto-plastic contact problems, International Journal of Solids and Structures, Vol. 39 (2002), pp. 2323-2341.

(14) Facchinei, F. and Pang, J.S., Finite-Dimensional Variational Inequalities and Complementarity Problems: Volume II, (2003), Springer-Verlag, New York.

(15) Website of SPOOLES, available from $<$ http://www.netlib.org/linalg/spooles/spooles.2.2.html > (accessed 2008-8-16). 